\title{
Sach-Register für Band XLIII
}

Die îett gedruckten Zahlen bezeichnen Original-Artikel. Bz. $=$ Bachanzeigen. P. $=$ Personalien. A.

Alkaliwirkung, konzentrierte auf

das Auge. 176. Amaurose, Behandlung der hyste-

ríschen durch Wachsuggestion und

Hypnose. 47. Amblyopie, Wirkung des Lichtes

bei toxí seller. 73 .

- $\quad$ Behandlung der hysterischen durch

Wachsuggestion und Hypnose. 47. Arteria centralis retinae, Em-bolie der. $4 * 21$.

- Thrombose der. 435.Astigmatismus, Veränderlichkeit

des Hornhaut-A. 144. Augenbewegungeu, Beziehungen

zwischen Sehen und. 249. Augenmuskeln, psychogene Stö-

rungen deräußeren im Kriege. 282. Augenverletzungen durch Selbst-

schüsse. 414. Au sf 1 ockungsmethode, Sachs-

Georgische. 609.

B.

Brille, eine alte. 172 .

C.

Cyklitis, Beziehungen der Iritis serosa zur. 625.

E.

Éisensplitter, 30 Jahre nach der

Verletzung aus der Regenbogen-

haut entfernt. 571. Ektropium, angeborenes des Ober-

lides 565. Elliotsche Operation. 355,377. Entzündungen, Sehstörungen in-

folge orbitalér. 438.

Erblindung ohne nachweisbare Ver-änderung am optischen Apparate 92.

Exoph thalmus, pulsierender. 534.

F.

Farbensinnstörungen, messende

Untersuchung der. 28. Pazialis, doppelseitige Lähmung des. 553.

- $\quad$ Verletzungen des. 524.Fremdkörper, B, bntgenlokalisation

intraokularer mit dem Stumpf-schen Apparat. 402.

G.

G-laukom, Beziehungen der Iritis serosa zum. 625.

selbständiges akutes iritisches. 339.

druckherabsetzende Wirkuug der

Myotika beim. 381. Grlaukomoperation. 333, 315, 355. 377.

$\mathrm{H}$. 
Herpes zoster uveae, Klinik und pathologische Anatomie des. 450.

$\mathrm{H}$ o r n h a n t, sy philitischer Primär-affekt an der. 123.

Hornhautastigmatismus, Veränderlichkeit des. 144.

Hypnose bei hysterischen Gesichtsstörungen. 47.

Hysterische Gesichtsstörungen. 47.

Infektionen des Bulbus, bak-terioskopische Prophj $1 / 8 x$ xe der post-operativen. 309.

Iritis serosa. 625.

Zeitschriit für Augenheilkundo. Bd. XLIV. Heft 6/6 23

850

Sach-Register für Band XLIII.

K.

Kalktherapie. 86. Knochengeschwülste dor Augen-

höhle. 216. Krebsmetastase im Hinterhaupts-

bein, doppelseitige Stauungspapille

bei. 23.

L.

L i c h t , Wirkung des bei den toxisehen Amblyopien. 78.

M.

Melanotische Creschwülste.

201. Metastasen iin Auge. 428. Milcheinspritzungen. 100. Mukozele deГ Keübeinhöhle und hinteren Siebbeinzelleu mit Atro-

phie der Sehnerven. 223. Myotika, d uckherabsetzende Wir-

kung der beim G-laucoma simplex.

381 .

$\mathrm{N}$.

Nachtblindheit, Behandlung der hysterischen durch Waehsug-gestion und Hypnose. 47.

Nystagmus det· Bergleute, Ent-stehung des. 264.

0 .

Ophthalmie, metastatische. 428. Orientierungsnntersueliungen,

ätiologische des praktischen Augen-

arztes. 579.

P.

Ptosis trachomatosa, Entstehung

der. 305. Pu pillar mem bran, bewegliche ent-

zü口dliche. 69.

It.

Regenbogenhaut, ein 30 Jalire

nach der Verletzung aus der R.

entfernter Eis $\theta d$ splitter. 571. Ringskotome im Telegraphen-

dienst. 681. Röntgeniok alisation intraoku-

larer Eremdkörper mit dem Stumpf-

schen Apparat. 402.

S.

Säurewirkung, kouzentrierte auf das Auge. 176.

Schädelverletzung und Sehner- 
venschwund. 687. Schwef elwasser stof $\mathrm{f}$, sekundäre Augenentzündungen durch. 195. Sehen und Augenbewegungen. 249. Sehnervenatrophie bei Mukozele der Keübeinhöhle und hinteren Siebbeinzellen. 223.

- $\quad$ bei Schädelverletzung. 687.Sehtheoriender griechischen Philosophen. 1. Selbstschüsse, Augenverletzungen durch, 411. Sklerodermie in Verbindung mit Star. 640. Spaltlam penmikroskopie, Tiefenlokalisation in der. 393. Star in Verbindung mit Sklerodermie. 640. Staroperation des Anfängers. 574.

- $\quad$ operative Behandlung der eitrigen Infektionen nach. 321. Stauungspapille, doppelseitige bei eiuer Krebsmetastase im Hinterhauptsbein. 23. Syphilis, Primäraffekt an der Hornhaut. 123.

T.

Telegraphendienst, Ringskotome im. 681. Tiefenlokalisation in der Spaltlampenmikroskopie. 393. Trachom, Entstehung der Ptosis trachomatosa. 30õ.

- $\quad$ chirurgische Behandlung des in Ägypten. 129. Träneuableitungsorgane, Er-

krankungen der. 242. Trigeminus, Verletzungen des.

524.

ü.

Ulcus corneae rodens. 480.

IV.

W a c h s t u m, Korrelationen des 654. Wa chsuggestion, bei hysterischen GesichtsstöruDgen. 47. Wirbelvenen des Auges. Druck in den. 141 .

$\mathrm{Z}$ i rkulati onsstör unge $\mathrm{n}$ des Auges. 421.

Namen-Register ftir Band XLIII.

851

N. Namen-Register îür Banò XLIII. 